\title{
artigo
}

Mendes, E.N.; Fiquene, J.C.; Bessani, L.; Santos Silva, F.C.M.; Máximo, N.F.; Alvarez, L.S.;

Hábitos de vida e consumo de alimentos pós pandemia de COVID-19 em São Luís, Maranhão, Brasil

\section{Hábitos de vida e consumo de alimentos pós pandemia de COVID-19 em São Luís, Maranhão, Brasil}

\author{
Life habits and food consumption after a COVID-19 pandemic in São Luís, Maranhão, Brazil \\ Hábitos de vida pos pandémicos y consumo de alimentos de COVID-19 en São Luís, Maranhão, Brasil
}

\begin{abstract}
RESUMO
Objetivo:Descrever os hábitos de vida e consumo de alimentos durante pandemia de COVID-19 em São Luís, Maranhão, Brasil. Método:Estudo transversal, descritivo e abordagem quantitativa. Foi realizado com moradores da região metropolitana de São Luís. A coleta de dados foi iniciada após consentimento do participante e assinatura online do Termo de Consentimento Livre e Esclarecido. Resultados:Participaram 349 pessoas, sexo feminino (71,35\%), idade média 29+10,8 anos.Quanto aos hábitos de vida os relatos foram: etilismo $(54,44 \%)$, tabagismo $(4,01 \%)$ e atividade física( $71,92 \%)$. A presença de COVID-19 foi relatado em $7,74 \%$ dos participantes, já $27,51 \%$ referem suspeita. 0 consumo de nutrientes para imunidade antes da pandemia era de $56,16 \%$, com o inicio da pandemia passou a 71,63\%. Os mais consumidos foram vitaminas C e D, limão e seus derivados. Conclusão:Conclui-se que 0 surgimento da pandemia aumentou a procura por nutrientes e alimentos que possam atuar no sistema imunológico.
\end{abstract}

DESCRITORES: SARS-CoV-2;Pandemia; Qualidade de vida.

\section{ABSTRACT}

Objective: To describe life habits and food consumption during the COVID-19 pandemic in São Luís, Maranhão, Brazil. Method: Cross-sectional, descriptive study and quantitative approach. It was carried out with residents of the metropolitan region of São Luís. Data collection started after the participant's consent and online signature of the Free and Informed Consent Form. Results: 349 people participated, female $(71,35 \%)$, mean age $29+10,8$ years. As for life habits, there ports were: alcoholism $(54,44 \%)$, smoking $(4,01 \%)$ and physical activity $(71,92 \%)$. The presence of COVID-19 was reported in $7,74 \%$ of the participants, while $27,51 \%$ reported suspicion. The consumption of nutrients for immunity before the pandemic was $56,16 \%$, with the beginning of the pandemic it increased to $71,63 \%$. The most consumed were vitamins $C$ and $D$, lemon and its derivatives. Conclusion: It is concluded that the emergence of the pandemic increased the demand for nutrients and foods that can act on the immune system.

DESCRIPTORS: SARS-CoV-2; Pandemic; Quality of life

\section{RESUMEN}

Objetivo:Describir hábitos de vida y consumo de alimentos durante la pandemia de COVID-19 en São Luís, Maranhão, Brasil. Método:Estudio transversal, descriptivo y abordajecuantitativo. Se llevó a cabo con residentes de laregión metropolitana de São Luís La recolección de datos se inicióconelconsentimientodel participante y la firma en línea delFormulario de Consentimiento Libre e Informado. Resultados:participaron 349 personas, mujeres (71,35\%), edad media 29 + 10,8 años. Encuanto a los hábitos de vida, los reportes fueron: alcoholismo $(54,44 \%)$, tabaquismo $(4,01 \%)$ y actividad física $(71,92 \%)$. La presencia de COVID-19 se informóenel $7,74 \%$ de los participantes, mientras que el $27,51 \%$ informósospecha. El consumo de nutrientes para lainmunidad antes de la pandemia fuedel $56,16 \%$, conelinicio de la pandemia aumentó a $71,63 \%$. Los más consumidos fueronlas vitaminas C y D, ellimón y sus derivados. Conclusión: Se concluye que laaparición de la pandemia incrementóla demanda de nutrientes y alimentos que puedenactuar sobre el sistema inmunológico.

DESCRIPTORES: SARS-CoV-2; Pandemia;Calidad de vida.

RECEBIDO EM: 18/09/2020 APROVADO EM: 28/09/2020

\section{Eliakim do Nascimento Mendes}

Coordenador do Curso de Nutrição do Centro Universitário UNDB, Mestre em Ciências da Saúde, Nutricionista.

ORCID: 0000-0002-9083-1240 
Joseane Costa Fiquene

Discente do Curso de Nutrição do Centro Universitário UNDB

ORCID: 0000-0003-3221-7549

\section{Lorena Bessani}

Discente do Curso de Nutrição do Centro Universitário UNDB

ORCID: 0000-0002-0896-2285

Fernanda Conceição Mota dos Santos Silva

Discente do Curso de Nutrição do Centro Universitário UNDB

ORCID: 0000-0003-3233-9482

Nathália Fernandes Máximo

Discente do Curso de Nutrição do Centro Universitário UNDB

ORCID: 0000-0002-0905-318X

\section{Luciana Silva Alvarez}

Discente do Curso de Nutrição do Centro Universitário UNDB

ORCID: 0000-0002-2707-6334

\section{INTRODUÇÃO}

0 COVID-19 (SARS-CoV-2) é da família dos coronavírus e são causadores de infecções respiratórias graves. O primeiro caso da doença é relatado em Wuhan, na China em 31 de dezembro de 2019. ${ }^{1}$ Desde janeiro de 2020, a Organização Mundial da Saúde (OMS) classificou o surto de COVID-19 como uma Emergência de Saúde Pública Internacional e, em março desse mesmo ano, uma pandemia mundial ${ }^{3}$. Outras entidades de saúde do Brasil e da América do Sul concordam que a pandemia é uma preocupação grave para a população brasileira. ${ }^{2,3}$

A disseminação do COVID-19 ocorre, principalmente, por contato entre indivíduos como: aperto de mão, saliva, espirro, tosse e catarro. Porém, pode haver contaminação por objetos ou superfícies como celulares, mesas, maçanetas, brinquedos, teclados de computador etc. ${ }^{4} \mathrm{O}$ diagnóstico é realizado por profissional de saúde que avalia sinais e sintomas e encaminha para analise biológica molecular (RT-PCR em tempo real) ou imunológico (teste rápido). ${ }^{5} \mathrm{Na}$ impossibilidade de realizar estasanálises, o diagnóstico pode ser feito considerando histórico de contato próximo ou domiciliar, nos últimos 7 dias antes do aparecimento dos sintomas, com caso confirmado laboratorialmente para COVID $-19 .^{6}$
Com o avento da

pandemia no Brasil

tem-se pesquisado

alternativas para

prevençãodo

COVID-19, dentre

elas a alimentação e

os hábitos de vida. $\mathrm{O}$

uso inadequado de

alimentos, nutrientes

e suplementos

vitamínicos pode

trazer consequências

para a saúde.
Segundo a OMS cerca de $80 \%$ dos contaminados pelo COVID-19 não apresentam nenhum sintoma, porém $20 \%$ podem precisar deacompanhamento hospitalar, sendo que $5 \%$ pode evoluir para forma mais grave da doença (necessitando de suporte ventilatório). ${ }^{2}$ Ainda não há tratamento com eficácia garantida para a doença. A prevenção tem sido o isolamento social recomendado por diversas entidades de saúde. ${ }^{2,3,6} \mathrm{~A}$ alteração abrupta da rotina pode ocasionar mudança dos hábitos de vida como etilismo, tabagismo e alimentação.

A alimentação saudável é essencial para prevenção e tratamento de diversas patologias. Com o avanço global da pandemia a alimentação passou a ser foco de estudos de associação em tratamentos do COVID-19. No entanto, muitas informações incorretas e/ou sem comprovação cientifica, também chamadas de fake News, são amplamente difundidas na população brasileira. Convém ressaltar que a alimentação é fundamental para manutenção da saúde e imprescindível para o sistema imunológico. ${ }^{7,8}$

Com o avento da pandemia no Brasiltem-se pesquisado alternativas para prevençãodo COVID-19, dentre elas a alimentação e os hábitos de vida. O uso inadequado de alimentos, nutrientes e suplementos vitamínicos pode trazer consequências para a saúde. ${ }^{9}$ É imprescindível identificar as alterações no cotidiano 
proporcionadas pelo COVID-19. Desde o início da pandemia de COVID-19, estratégias de saúde têm sido amplamente orientadas: prática de atividade física, diminuição do etilismo e tabagismo e alimentação adequada. $\mathrm{O}$ objetivo do estudo foidescrever os hábitos de vida e consumo de alimentos durante pandemia de COVID-19 em São Luís, Maranhão, Brasil.

\section{MÉTODOS}

Trata-se de um estudo transversal, quantitativo de abordagem descritiva que visa avaliar os impactos nos hábitos de vida e consumo de nutrientes durante pandemia de COVID-19. A coleta dos dados foi realizada no mêsde maio de 2020 com moradores da Região metropolitana de São Luís, que inclui os municípios de São Luís, Raposa, Paço do Lumiar e São José de Ribamar no Maranhão, Brasil. Toda a pesquisa foi realizada de maneira virtual respeitando as medidas de isolamento social recomendadas pelas entidades de saúde.

A amostra se deu de forma não probabilística, por conveniência. O critério de inclusão adotado foram moradores da região metropolitana de São Luís e que possuíam acesso a dispositivo eletrônico com acesso a internet para resposta do questionário on-line. Os critérios de exclusão foram pessoas em imunoterapia por tratamento oncológico ou pessoas em processo de internação hospitalar ou aquelas com recusa a participação no estudo.

O recrutamento da amostra se deu de forma on-line nas redes sociais, onde foi apresentado imagem/convite para participação do estudo. A pesquisa foi amplamente divulgada pelos pesquisadores nas redes sociais (Instagram e Facebook) e aplicativos de mensagens (WhatsApp) para busca de candidatos. Os indivíduos considerados elegíveis foram convidados a participar do estudo. Em seguida, foi encaminhado o link de acesso a planilha eletrônica do Google Forms, previamente editada pelo grupo de pesquisa. A tela de início da planilha continha o Termo de Consentimento Livre e Esclarecido (TCLE) com linguagem clara e acessível e, virtualmente, deveria ser assinado dando concordância a participação voluntária no estudo. Após consentimento foi enviado por e-mail uma via assinada virtualmente do TCLE ao pesquisado.

Os participantes responderam o questionário eletrônico que continha questões abertas e fechadas. Os dados avaliados foram sociodemográficos, hábitos de vida, situação de saúde e de alimentação. Os dados sociodemograficos avaliados foram idade, sexo, e cidade de moradia. Quanto aos hábitos de vida avaliou-se etilismo, tabagismo e atividade física. Etilismo e tabagismo foram avaliados quanto a presença de consumo antes da pandemia, e quando presente se aumento, diminuição e manutenção de consumo após a pandemia. Não
Tabela 01.Perfil sociogeográfico de moradores da região metropolitana de São Luís durante pandemia de COVID-19.
Variável

Idade (média+DP)

Sexo - n (\%)

Cidade atual - n (\%)
$29,31+10,8$

$\begin{array}{cc}\text { Feminino } & 249(71,35 \%) \\ \text { Masculino } & 100(28,65 \%) \\ \text { São Luís } & 318(91,12 \%) \\ \text { José de Ribamar } & 22(6,30 \%) \\ \text { Paço do Lumiar } & 07(2,01 \%) \\ \text { Raposa } & 2(0,57 \%)\end{array}$

$3,65+1,47$ foi avaliado o nível de atividade física, avaliou-se apenas a presença ou ausência de alguma atividade física antes da pandemia e se após o inicio houve aumento ou diminuição da frequência de atividade física.

A situação de saúde foi avaliada pelo relato de comorbidades e presença de $\mathrm{CO}$ VID-19 no pesquisado ou em morador da mesma residência. Quanto a alimentação avaliou-se o consumo de alimentos, nutrientes e vitaminas antes e durante a pandemia. A pergunta relacionada ao consumo de nutrientes foi aberta, possibilitando ao pesquisado inserir quantos alimentos/ nutrientes utilizou para imunidade antes e durante a pandemia.

Os dados foram tabulados no programa Excel Software Microsoft. As variáveis numéricas serão apresentadas em média e desvio padrão e as categóricas em números absolutos e relativos. A análise estatística foi realizada pelo programa Stata versão 14.0, sendo considerado estatisticamente significante quando $\mathrm{p}<0,05$. Para análise de associação das variáveis categóricas utilizou-se o teste de quiquadrado. Para análise das variáveis numéricas o teste $t$ de student.

Este projeto foi iniciado após submissão e aprovação do Comitê de Ética em Pesquisa do Centro Universitário UNDB (CEP-UNDB), sob número de parecer 4.046 .556 e CAAE 32225120.0.0000.8707. Foram respeitados todos os preceitos éticos de pesquisa conforme determina a Resolução do Conselho Nacional de Saúde, Resolução 466/2012.

\section{RESULTADOS}

Participaram do estudo 349 pessoas com idade média de 29,31+10,8 anos com predomínio de sexo feminino $(71,35 \%)$, conforme tabela 01. A região metropolitana de São Luís é formada por 4 municípios (São Luís, São José de Ribamar, Paço do Lumiar e Raposa). A capital do Maranhão, São Luís, apresentou o maior número de participantes $(91,12 \%)$. Avaliou-se a quantidade de moradores em uma mesma residência incluindo o pesquisado e o resultado foi em média, $3,65+1,47$ pessoas por domicílio. 
Tabela 02. Etilismo, tabagismo e prática de atividade física de moradores da região metropolitana de São Luís durante pandemia de COVID-19

$\begin{array}{lccc}\text { Variável } & \mathrm{n}(\%) & \text { p valor } \\ \text { 1.Etilismo } & 190(54,44 \%) & 0,137 \\ \text { 1.1Durante a pandemia o etilismo: } & \text { Aumentou } & 21(11,05 \%) & \\ & \text { Diminuiu } & 123(64,74 \%) & 0,143 \\ & \text { Indiferente } & 46(24,21 \%) & \\ \text { 2.Tabagismo } & 14(4,01 \%) & 0,269 \\ \text { 2.1 Durante a pandemia o tabagismo: } & \text { Aumentou } & 3(21,43 \%) & \\ & \text { Diminuiu } & 5(35,71 \%) & 0,453 \\ \text { 3. Prática de Atividade Física } & \text { Indiferente } & 6(42,86 \%) & \\ \text { 3.1Durante a pandemia o nível de AF: } & \text { Aumentou } & 57(22,89 \%) & 0,110 \\ & \text { Diminuiu } & 142(57,03 \%) & 0,299\end{array}$

Tabela 03. Histórico de saúde de moradores da região metropolitana de São Luís durante pandemia de COVID-19.

\begin{tabular}{|c|c|c|}
\hline Variável & & p valor \\
\hline 1.Presença de Comorbidades - $\mathrm{n}(\%)$ & $65(18,62 \%)$ & 0,597 \\
\hline 1.1 Diabetes- $n(\%)$ & $4(6,15 \%)$ & 0,597 \\
\hline 1.2 Hipertensão- n (\%) & $13(20,00 \%)$ & 0,796 \\
\hline 1.3 Problemas Cardíacos- n (\%) & $4(6,15 \%)$ & 0,597 \\
\hline 1.4 Dislipidemia- n (\%) & $13(20,00 \%)$ & 0,796 \\
\hline 1.5 Obesidade- $\mathrm{n}(\%)$ & $11(16,92 \%)$ & 0,351 \\
\hline 1.6 Doenças Respiratórias- n (\%) & $12(18,46 \%)$ & 0,728 \\
\hline 2. Diagnóstico de COVID-19- n (\%) & $27(7,74 \%)$ & 0,000 \\
\hline 2.1 Média de dias de sintomas-(média+DP) & \multicolumn{2}{|c|}{$9,15+6,72$} \\
\hline 3. Suspeita de COVID-19 - n (\%) & $96(27,51 \%)$ & 0,276 \\
\hline 3.1 Média de dias sintomas-(média+DP) & \multicolumn{2}{|c|}{$8,58+6,40$} \\
\hline 4. Morador da residência com COVID- $\mathrm{n}(\%)$ & $59(16,91 \%)$ & 0,001 \\
\hline Fonte: Elaborada pelos autores, 2020. & & \\
\hline
\end{tabular}

Tabela 04. Consumo de nutrientes para imunidade por moradores da região metropolitana de São Luís durante pandemia de COVID-19.

Variável

$n(\%)$

p valor

Consumo de nutrientes para imuni-

dade antes da pandemia.

$196(56,16 \%) \quad 0,002$

Consumo de nutrientes para imunidade com o início da pandemia.

$250(71,63 \%)$

0,461

Nutrientes Consumidos.

$\begin{array}{lll}\text { Vitamina C } & 277(79,37 \%) & 0,203 \\ \text { Vitamina D } & 167(47,85 \%) & 0,665 \\ \text { Limão } & 217(62,18 \%) & 0,481\end{array}$

Durante a pandemia de COVID-19 os hábitos de vida podem ser influenciados significativamente. Dos participantes do estudo $54,44 \%$ são etilistas. Ao avaliar se a pandemia provocou mudanças no etilismo $11,05 \%$ relatam que aumentaram o consumo de bebida alcoólica (tabela 02). O tabagismo esteve presente em 4,01\% dos avaliados e destes, $42,86 \%$ informam que o hábito de fumar não sofreu alteração com a pandemia. A atividade física foi relatada como frequente em $71.92 \%$ dos participantes, porém $57,03 \%$ dos que praticam atividade informaram que o nível de atividade física diminuiu com a pandemia.

O histórico de saúde tem sido um importante aliado no combate ao novo coronavírus, porém a presença de comorbidades representa riscos significativos. A presença de pelo menos uma comorbidade foi referida em $18,62 \%$ dos participantes do estudo (tabela 03). As comorbidades mais frequentes foram: hipertensão e dislipidemia, ambas com 20,00\% cada. As doenças respiratórias como asma, sinusite, rinite e outras foi relatada por $18,46 \%$.

Somente o exame pode confirmar a presença de COVID-19, nesse estudo 7,74\% referiramtestar positivo para COVID-19. O tempo médio, em dias, de apresentação dos sintomas foi de 9,15+6,72. Avaliou-se ainda os participantes que suspeitam de contaminação por COVID-19 por ter apresentando algum sintoma característico, porém sem diagnóstico por exame. Os dados apontam que $27,51 \%$ suspeitam de contaminação e o tempo médio de sintomas foi de $8,58+6,4$ dias.

Neste estudo foi avaliado se o participante fazia uso de algum nutriente para imunidade antes da pandemia de COVID-19 e 56,16\% disseram que sim, conforme a tabela 04 . Com o inicio da pandemia, o percentual aumentou alcançando a marca de 71,63\%.

Quanto aos nutrientes mais comuns de uso, os mais frequentes foram: vitamina C $(79,37 \%)$, limão e seus derivados $(62,18 \%)$, vitamina D $(47,85 \%)$ e complexos vitamínicos/multivitamíni$\cos (42,41 \%)$. A indicação ou prescrição desses nutrientes precisa ser feita por um 


\begin{tabular}{llcr} 
& Alho & $87(24,93 \%)$ & 0,293 \\
& Glutamina & $38(10,89 \%)$ & 0,009 \\
& Prebioticos/probióticos & $43(12,32 \%)$ & 0,007 \\
Nutrientes Consumidos. & Mel de Abelha & $129(36,96 \%)$ & 0,993 \\
& Gengibre & $94(26,93 \%)$ & 0,435 \\
& Propolis & $107(30,66 \%)$ & 0,040 \\
& Multivitamínicos & $148(42,41 \%)$ & 0,150 \\
& Médico & $65(22,29 \%)$ & \\
Quem prescreveu/indicou & Nutricionista & $31(10,73 \%)$ & \\
nutriente (s)? & Amigo(a) & $20(6,92 \%)$ & 0,569 \\
& Familiar & $113(39,10 \%)$ & \\
& Internet & $32(11,07 \%)$ & \\
Fonte: Elaborada pelos autores, 2020. & Outros & $28(9,69 \%)$ & \\
\hline
\end{tabular}

profissional de saúde qualificado. Neste estudo, a maioria dos participantes $(39,10 \%)$ recebeu indicação de familiar, seguido de busca na internet $(11,07 \%)$ para uso dos nutrientes.

\section{DISCUSSÃO}

O crescimento acelerado dos casos de COVID-19 no Brasil ${ }^{6}$ e no mundo ${ }^{2}$ tornou a doença um problema de saúde pública internacional. A média de idade deste estudo foi de 29,31+10,8 anos, um público jovem. No entanto, a população idosa é considerada mais suscetível a complicações pelo COVID-19. ${ }^{10}$ Os idosos tem maior tempo de internação hospitalar, maior necessidade de intubação e desfechos clínicos desfavoráveis como o óbito. ${ }^{11}$ Além da longevidade,é necessário considerar os hábitos de vida como etilismo, tabagismo e a prática de atividade física.

O isolamento social recomendado por entidades de saúde ${ }^{2,3,6}$ pode levar a distúrbios como alcoolismo ${ }^{12}$, tabagismo ${ }^{13} \mathrm{e}$ sedentarismo ${ }^{14}$. O consumo de álcool anterior a pandemia foi relatado por $54,44 \%$ (190) dos participantes desse estudo, cerca de 35,00\% destes referem que aumentaram ou mantiveram o mesmo consumo. $\mathrm{O}$ consumo de álcool, principalmente em altas doses, aumenta o risco de infecção grave por COVID-19 devido comprometimento imunológico, relação com comorbidades,

\section{Dos praticantes}

de atividades

física apresentados

nesse estudo

cerca de $\mathbf{5 7 , 0 0 \%}$

referem ter

diminuído a

prática com

o início da

pandemia. efeito deletério no fígado, descompensação psicológica e outros. ${ }^{12}$

Além do consumo de álcool, o tabagismo tem sido uma grande preocupação. Apesar do baixo índice de tabagismo presente neste estudo (4,01\%), as pessoas que fumam ou as que convivem com fumantes são vulneráveis a diversas patologias, incluindo o COVID-19. Os riscos estão relacionados a saúde respiratória já comprometida nesse público e exacerbada frente a infecção por COVID-19 portanto, podem aumentar o risco de óbito. ${ }^{13}$ A saúde respiratória pode ser melhorada com a prática de atividade física.

Dos praticantes de atividades física apresentados nesse estudo cerca de $57,00 \%$ referem ter diminuído a prática com o início da pandemia. A redução da prática pode ser associada ao isolamento social que diminuiu o acesso aos centros de atividades física e as atividades realizadas ao ar livre. ${ }^{14}$ Quase metade da população em estudo refere sedentarismo. A OMS possui recomendações especificas de atividade física para a população. ${ }^{2}$

A presença de comorbidade é fator de risco para infecção por COVID-19.13 Cerca de $18,00 \%$ dos participantes deste estudo informaram pelo menos uma, sendo as mais prevalenteshipertensão, dislipidemia, doenças respiratórias, obesidade e diabetes. Em metanalisepublicada recentemente, que avaliou a presença de comorbidade em 1576 pacientes acometidos por COVID-19, as mais prevalentes foram hipertensão (21,1\%, IC 95\%: 13,0-27,2\%) e diabetes (9,7\%, IC 95\%: 7,2-12,2\%), seguidas por doença cardiovascular $(8,4 \%$, IC 95\%: 3,8-13,8\%) e doença do sistema respiratório (1,5\%, IC 95\%: 0,9-2,1\%) ${ }^{15}$.

O Brasil, hoje 02 de junho de 2020, já ultrapassou mais de 500 mil casos confirmados de coronavirus6. Porém, acredita-se que o número de caso seja subnotificado, sendo o principalmente motivo a ausência de testes disponíveis a população. ${ }^{16} \mathrm{~A}$ suspeita de contaminação foi referida por $27,51 \%$ dos participantes. Essa suspeita parte da apresentação de sintomas no período de pandemia. Os principais sintomas da COVID-19 são 
febre, tosse seca, cansaço, dores e desconforto, dor de garganta, diarreia e outros. ${ }^{17} \mathrm{O}$ tempo médio de sintomas relatados no estudo em casos confirmados e suspeitos são $9,15+6,72$ e $8,58+6,40$ dias, respectivamente. $\mathrm{O}$ tempo médio de sintomas pode chegar a 11,5 dias. ${ }^{18}$ Para minimização dos sintomas e, principalmente, para evitar a COVID-19 estratégias de alimentação são sugeridas por especialistas. ${ }^{7}$

Durante a pandemia, é de grande importância os cuidados com alimentação, especialmente no que diz respeito aos excessos e as restrições que possam comprometer o sistema imunológico. ${ }^{19}$ Este estudo revelou que houve um aumento no consumo de alimentos "para imunidade" com o inicio da pandemia e que o consumo de nutrientes, antes da pandemia, tem relação com os casos confirmados de COVID-19. Os alimentos/nutrientes mais consumidos foram Vitamina C (vit. C), limão, Vitamina $\mathrm{D}$, Complexos vitamínicos e mel de abelha.

O consumo de vit $\mathrm{C}$ mostrou-se majoritário, seja em sua forma isolada ou com o limão, rico nesse nutriente. A vit. C é um antioxidante capaz de aumentar os subconjuntos das células $\mathrm{T}$, melhora a resposta linfocitária e aumenta a produção de interleucina-2, portanto com ação na função imunológica. ${ }^{20}$ Quase $50,00 \%$ dos participantes desse estudo referem consumo de vitamina D. A vitamina $\mathrm{D}$ pode reduzir o risco de infecções, reduzir as taxas de replicação viral e reduzir as concentrações de citocinas pró-inflamatórias, além de aumentar as concentrações de citocinas anti-inflamatórias. ${ }^{21}$ Evidências recentes apontam que a suplementação de vitamina $\mathrm{D}$ pode reduzir o risco de infecções e mortes por COVID-19. ${ }^{22}$ O consumo de complexos de vitaminas pode estar relacionado a busca por outros benefícios para imunidade proporcionado pela vitamina $\mathrm{E}$, zinco, selênio, dentre outros. ${ }^{20}$

O uso do mel de abelha e o própolis foi relatado por mais de 30,00\% dos participantes do estudo. $\mathrm{O}$ mel de abelha
Confirmamos que

houve um aumento

no consumo de

alimentos e nutrientes

pelos participantes

do estudo com

início da pandemia.

Esse consumo pode

trazer benefícios

significativos para o

sistema imunológico.

O consumo de

nutrientes antes da

pandemia, glutamina,

prebioticos/

probioticos e própolis

apresentaram

associação em

relação a presença

de COVID-19

confirmada por

exame. sem ferrão pode reduzir a gravidade de manifestações pulmonares em infecções por COVID-19. ${ }^{23}$ Estudo recenteaponta o potencial dos flavonoides dos extratos etanolicos de própolis para tratamento do COVID-19.24

Apesar dos diversos benefícios de nutrientes e alimentos para a alimentação19, é importante ressaltar que quase $70,00 \%$ dos participantes da pesquisa não foi orientado por um profissional médico ou nutricionista. Porém entidades de saúde e nutrição reforçam a importância do autocuidado com a alimentação 7 . E, ainda, não há alimento ou nutriente com eficácia garantida para tratamento do novo coronavírus, o COVID-19.

\section{CONCLUSÃO}

A idade média dos participantes do estudo revelou um público adulto jovem, no entanto a popução mais vulnerável a infecção são os idosos. Outra situação de suscetibilidade a infecção por COVID-19 são os hábitos de vida inadequados como etilismo, tabagismo e sedentarismo.

Além dos riscos do grupo com hábitos de vida ruins, é importante atentar ao público com comorbidades, pois são associados a desfechos clínicos desfavoráveis. A diabetes, hipertensão, problemas cardíacos e doenças respiratória aumentam o risco de óbito, e foram as mais presentes nesse estudo.

Confirmamos que houve um aumento no consumo de alimentos e nutrientes pelos participantes do estudo com início da pandemia. Esse consumo pode trazer benefícios significativos para o sistema imunológico. O consumo de nutrientes antes da pandemia, glutamina, prebioticos/probioticos e própolis apresentaram associação em relação a presença de $\mathrm{CO}$ VID-19 confirmada por exame.

Em virtude das limitações temporais e da impossibilidade de contato direto com o pesquisado para respostas do questionário, outros estudos são necessários para esclarecer as questões que esta pesquisa levantou. 


\section{REFERÊNCIAS}

1. Chen N, Zhou M, Dong X, Qu J, Gong F, Han Y, et al.Epidemiological and clinical characteristics of 99 cases of 2019 novel coronavirus pneumonia in Wuhan, China: a descriptive study. The Lancet. 2020; 395(10223):513-507. https://doi. org/10.1016/S0140-6736(20)30211-7

2. World Health Organization - WHO. Corona virus disease 2019. Geneva: World Health Organization; 2020 [acesso 29 mai 2020]. Disponivelem: https://www.who.int/ emergencies/ diseases/novel-coronavirus-2019/events-as-they-happen

3. Pan-American Health Organization - PAHO. OMS afirma que COVID-19 é agora caracterizada como pandemia. Washington: Pan-American Health Organization, 2020[acesso 29 Mai 2020]. Disponível em: https://www.paho.org/en/documents/ongoing-living-update-potential-COVID-19-therapeutics-summary-rapid-systematic-reviews

4. Rothan HA, Byrareddy SN. The epidemiology and pathogenesis of coronavirus disease (COVID-19) outbreak. J Autoimmun. 2020;109:102433. https://doi.org/10.1016/j. jaut.2020.102433

5.Quintella CM, Quintella HM, Palma GB, Silva SCR, Silva GHR. Coronavírus (sars-Cov-2) e COVID-19: mapeamento de testes clínicos. Cadernos de prospecção. 2020; 13(2): 411-397.doi: https://dx.doi.org/10.9771/cp.v13i2.COVID-19.36175

6. Ministério da Saúde (BR). Coronavírus Brasil. Brasília: Ministério da Saúde; 2020 [acesso 29 Mai 2020]. Disponívelem: https://covid.saude.gov.br/

7. Associação Brasileira de Nutrição (ASBRAN). Guia para uma alimentação saudável em tempos de Covid-19. Brasília: ASBRAN; 2020 [acesso 29 Mai 2020]. Disponivel em: https:// www.asbran.org.br/storage/downloads/files/2020/03/ guia-alimentar-covid-19.pdf.

8. Campos LF, Barreto PA, Ceniccola GD, Gonçalves RC, Matos LBN, Zambelli CMSF, et al. Parecer BRASPEN/AMIB para enfrentamento do COVID 19 em pacientes Hospitalizados. BRASPEN J. 2020;35(1): 5-3. doi:https://doi.org/10.37111/ braspenj.parecerbraspen 2020

9. Madden JM, Shetty OS, Zhang F, Briesacher BA, Ross-Degnan D, Soumerai SB, et al. Risk Factors Associated With Food Insecurity in the Medicare Population. JAMA Intern Med. 2020;180(1):147-144. doi:https://doi.org/10.1001/jamainternmed.2019.3900

10. Liu K, Chen Y, Lin R, Han K. Clinical features of COVID-19 in elderly patients: A comparison with young and middle-aged patients. J Infect. 2020; 80(6):18-14. doi:https://doi. org/10.1016/j.jinf.2020.03.005

11. Liu W, Tao ZM, Lei W, Yuan ML, Liu K, Zhou L, et al. Analysis of factors associated with disease outcomes in hospitalized patients with 2019 novelcoronavirus disease. Chin Med J. 2020; 133(9):1038-1032. doi: https://doi.org/10.1097/ CM9.0000000000000775

12. Da BL, Im GY, Schiano. COVID-19 Hangover: A Rising Tide of Alcohol Use Disorder and Alcohol Associated Liver Disease. Hepatology. 2020. doi:https://doi.org/10.1002/hep.31307

13. Volkow ND. Collision of the COVID-19 and Addiction Epidemics. 2020. doi: https://doi.org/10.7326/M20-1212

14. Hammami A, Harrabi B, Mohr M, Krustrup P. Physical activity and coronavirus disease 2019 (COVID-19): specific recommendations for home-based physical training, Managing Sport and Leisure. doi: https://doi.org/10.1080/23750472.2020.1757494

15. Yang J, Zheng Y, Gou X, Pu K, Zhaofeng C, Guo Q, et al. Prevalence of Comorbidities and Its Effects in Patients Infected With SARS-CoV-2: A Systematic Review and Meta-Analysis. Int J Infect Dis. 2020; 94(1): 95-91. doi:https://doi.org/10.1016/j. ijid.2020.03.017.

16. Dias GH, Souza CDS, Bezerra MR, Peixoto FS. Análise da distribuição espacial da covid-19 e subnotificação de casos novos e óbitos no estado do Rio Grande do Norte, Brasil. Revista Pensar Geografia. 2020;3(2):67-51. doi: https://doi.org/10.26704/ rpgeo

17. Wimalawansa, S.J. Global epidemicofcoronavirus--COVID-19: Whatwecan do tominimzerisksl. Eur. J. Biomed. Pharm. Sci. 2020;7(3):438-432. https://www.ejbps.com/ejbps/ abstract_id/6656

18. Lauer AS, Grantz KH, Bi O, Jones F. The Incubation Period of Coronavirus Disease 2019 (COVID-19) From Publicly Reported Confirmed Cases: Estimation and Application. Ann Intern Med. 2020;172(9):582-577. doi: https://doi.org/10.7326/M20-0504

19. Muscogiuri G, Barrea L, Savastano S, Colao A. Nutritional recommendations for CoVID-19 quarantine. Eur J Clin Nutr.2020. doi:https://doi.org/10.1038/s41430-020-0635-2

20. Chandra RK. Effect of vitamin and trace-element supplementation on immune responses and infection in elderly subjects. Lancet. 1992;340(8828):1124-7. doi: https://doi. org/10.1016/0140-6736 (92) 93151-c.

21. Gruber-Bzura BM. Vitamin D and Influenza-Prevention or Therapy? Int. J. Mol. Sci. 2018;19(8):25-1. doi:https://doi. org/10.3390/ijms19082419

22. Grant WB, Lahore H, McDonnell SL, Baggerly CA, French CB, Aliano JL, et al. Evidence that Vitamin D Supplementation Could Reduce Risk of Influenza and COVID-19 Infections and Deaths. Nutrients. 2020;12(4):19-1. doi:https://doi.org/10.3390/ nu12040988

23. Mustafa MZ, Shamsuddin SH, Sulaiman SA, Abdullah JM. Anti-inflammatory properties of stingless bee honey may reduce the severity of pulmonary manifestations in COVID-19 infections. Malays J Med Sci. 2020;27(2):169-165. doi:https:// doi.org/10.21315/mjms2020.27.2.17

24.Guler HI, Tatar G, Yildiz O, Belduz AO, Kolayli S. Investigation of potential inhibitor properties of ethanolic propolis extracts against ACE-II receptors for COVID-19 treatment by Molecular Docking Study. ScienceOpen. 2020. doi:https://doi. org/10.14293/S2199-1006.1.SOR-.PP5BWN4.v1 